\title{
Adenoma negro de la glándula suprarrenal (Black Adenoma)*
}

\author{
Drs. OCTAVIO A. CASTILLO C. ${ }^{1,2}$, LEONARDO ARELLANO H. ${ }^{3}$
}

1 Unidad de Urología, Clínica INDISA.

2 Facultad de Medicina, Universidad Andrés Bello.

3 Unidad de Histopatología, Clínica AVANSALUD.

Santiago, Chile.

\section{Black adrenal adenoma}

Introduction: The black adenoma is a rare tumor of the adrenal gland. Clinical case: A male patient treated for lung carcinoma, was found to have an incidental adrenal mass. Due to a suspicious of metastatic disease, a laparoscopic adrenalectomy was done. The biopsy showed a black adenoma. After 11 years of follow-up, there is no evidence of recurrence. Conclusion: The black adenoma of the adrenal gland is a rare, benign and non-functioning tumor. The accurate diagnosis is done only by histological studies.

Key words: Incidentaloma, black adenoma, adrenal gland.

\section{Resumen}

Introducción: El adenoma negro de la glándula suprarrenal, o Black adenoma, es una patología de baja frecuencia dentro las masas suprarrenales. Caso clínico: Presentamos el caso de un paciente tratado por un Cáncer pulmonar, con el diagnóstico incidental de una masa suprarrenal izquierda que requirió extirpación laparoscópica por la sospecha de metástasis. La biopsia confirmó la presencia de un Adenoma negro. El paciente se encuentra vivo 11 años después. Conclusión: El adenoma negro es un tumor suprarrenal raro, benigno, no funcionante, cuyo diagnóstico es solamente histológico.

Palabras clave: Adenoma suprarrenal, metástasis, adenoma negro.

\section{Introducción}

Un incidentaloma suprarrenal es una masa mayor de $1 \mathrm{~cm}$, descubierta accidentalmente en estudios imagenológicos hechos por otras causas distintas de un estudio suprarrenal ${ }^{1}$. La mayoría corresponden a adenomas corticales, aunque también se han encontrado adenomas funcionantes, feocromocitomas, carcinoma suprarrenal y metástasis ${ }^{2-7}$.

Estudios en autopsia han determinado una fre-

*Recibido el 2 de marzo de 2013 y aceptado para publicación el 28 de marzo de 2013.

Los autores no refieren conflictos de interés.

Correspondencia: Dr. Octavio A. Castillo C. Avenida Santa María 1810, Santiago, Chile. P.C.: 7520440. octavio.castillo@indisa.cl 
cuencia de $6 \%$, con un rango de 1 a $31 \%$, y una incidencia de $0,2 \%$ en pacientes entre 20 y 29 años, y de $7 \%$ en mayores de 70 años, en estudios de Tomografía computada ${ }^{3}$.

El adenoma negro de la glándula suprarrenal, o Black-Adenoma (BA), no funcionante, es un raro caso de hallazgo en autopsias, y en pacientes con BA funcionante debe descartarse un patología maligna, dado la dificultad del diagnóstico diferencial, incluso con los métodos radiológicos actuales.

Este caso ilustra el hallazgo de una masa suprarrenal izquierda en un paciente con diagnóstico de cáncer pulmonar operado, razón por la cual se decidió realizar adrenalectomía laparoscópica ante la sospecha radiológica de una metástasis.

\section{Caso clínico}

Paciente de 61 años, con antecedente de litiasis urinaria recurrente y una lobectomía pulmonar izquierda en agosto de 2000 por cáncer de pulmón. En el seguimiento se pesquisa en la tomografía computada, a los 3 meses, una masa suprarrenal izquierda no visible en sus exámenes anteriores (Figura 1). El estudio endocrinológico es negativo para hiperfunción hormonal. Dado su antecedente de cáncer de pulmón y la imposibilidad de descartar una metástasis, se discuten las opciones de punción citológica o cirugía laparoscópica. Conocidos los riesgos y beneficios de cada procedimiento, el paciente opta por una adrenalectomía izquierda laparoscópica.

Se realiza una adrenalectomía laparoscópica izquierda con técnica de 3 trocares. El tiempo operatorio fue de $90 \mathrm{~min}$ con un sangrado estimado de $150 \mathrm{ml}$. Su postoperatorio fue sin incidentes siendo dado de alta a las $36 \mathrm{~h}$ de la cirugía.

La biopsia informa una muestra que corresponde a tejido adiposo y glándula suprarrenal izquierda, de $5 \mathrm{~cm}$ y $33 \mathrm{~g}$. Al corte presenta un nódulo bien delimitado, de superficie homogénea, pardo-negruzca que mide $2,7 \times 1,8 \mathrm{~cm}$ (Figura 2). Al examen microscópico el nódulo corresponde a proliferación de células de tamaño mediano, de citoplasma abundante eosinofílico granular, con abundante pigmento pardo amarillento (neuromelanina). Las células se disponen en lóbulos, alveolos, separadas por tabiques ricamente vascularizados, en algunas zonas las células presentan citoplasma claro, el núcleo es vesiculoso con nucléolo eosinófilo algo prominente. Se observa leve pleomorfismo celular y anisocitosis (Figura 3).

Luego de 11 años de seguimiento el paciente se encuentra sano, sin evidencia de recurrencia tumoral.

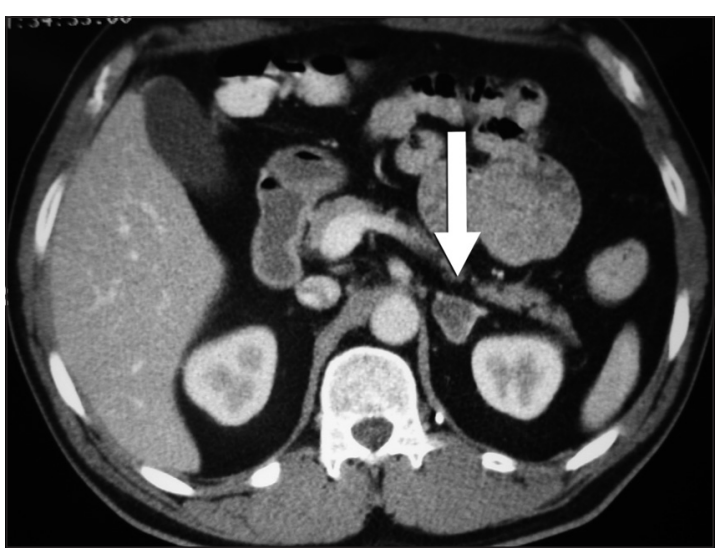

Figura 1. Tomografía computada que muestra nódulo suprarrenal, homogéneo, que capta medio de contraste, sin características de adenoma benigno.

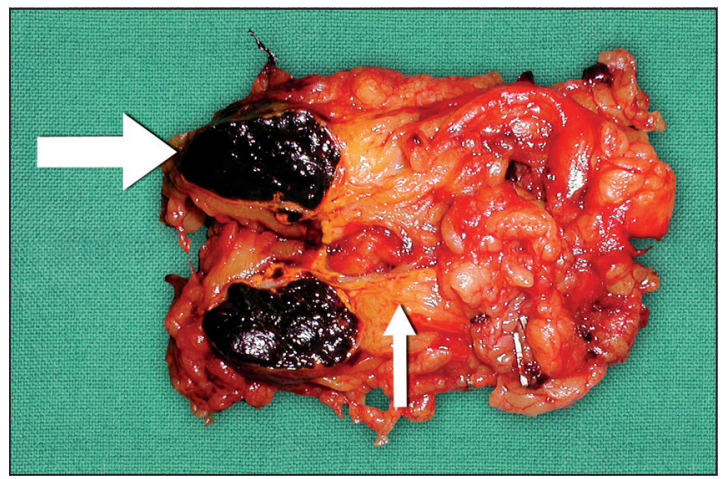

Figura 2. Corte macroscópico que muestra el adenoma negro (flecha ancha) y un asta de la glándula suprarrenal normal (flecha delgada).

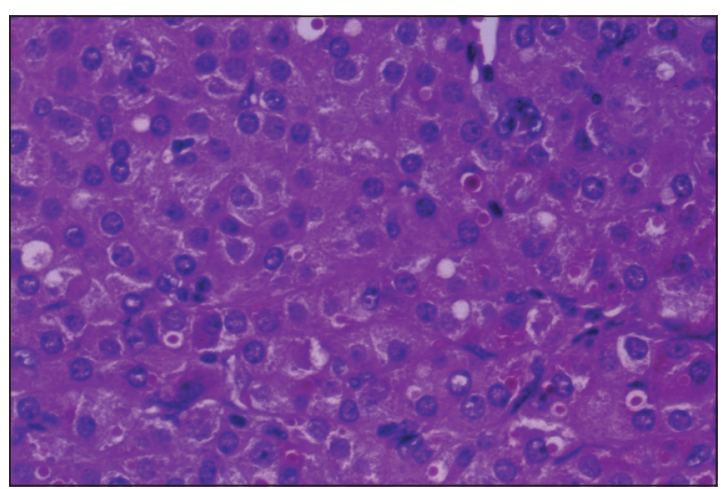

Figura 3. H.E. x 400. El tumor está compuesto por proliferación de células de tamaño mediano relativamente homogéneas de citoplasma eosinófilo con depósito de pigmento, núcleo ovoideo central sin gran pleomorfismo ni atipía. Las células se disponen en cordones en un estroma vascularizado. 


\section{Discusión}

Con el actual uso de la imagenología, la prevalencia de incidentalomas suprarrenales ha aumentado hasta un 5\%. Aunque la Tomografía computada (TC), Resonancia magnética (RM) MRI y el PET/ SCAN permiten caracterizar imagenológicamente la mayoría de los adenomas benignos, tanto ellas como la Cintigrafía suprarrenal MIGB, no pueden dar evidencia concluyente de su benignidad ${ }^{1}$. La glándula suprarrenal es un sitio común de metástasis ${ }^{8}$, por tanto, es mandatorio diferenciar metástasis de adenomas benignos para una adecuada estadificación en los pacientes con patología maligna, especialmente en el carcinoma pulmonar. El PET/CT es una herramienta de ayuda importante en esta diferenciación y puede ser de utilidad para diferenciar lesiones adrenales benignas de malignas?

No se han descrito guías clínicas que determinen la evaluación de los incidentalomas suprarrenales, por tanto, en general se siguen las indicaciones de la NIH publicada en el año $2003^{10}$.

La historia clínica y un examen físico completo son mandatorios, con el propósito de evaluar síntomas y signos de hipersecreción hormonal. Se recomienda realizar test de supresión con Dexametasona, Metanefrinas y Catecolaminas urinarias en $24 \mathrm{~h}, \mathrm{y}$ en caso de historia de hipertensión, Aldosterona y Renina plasmáticas para descartar un Hiperaldosteronismo. Si se trata de un adenoma funcionante está indicada la adrenalectomía laparoscópica. Si la TC es sospechosa de metástasis se puede realizar citología por punción (habiendo descartado se trate de un Feocromocitoma). Se recomienda seguimiento con TC a 6, 12 y 24 meses, y estudio hormonal anualmente si la lesión aparenta ser benigna y no funcionante. La cirugía en estos casos estaría indicada para tumores mayores de $4 \mathrm{~cm}$, si hay crecimiento en el control radiológico o si hay evidencia de hiperfunción hormonal.

El BA es un adenoma suprarrenal que tiene una apariencia café-oscura o negra, es pobre en lípidos y acumula Lipofucsina. Generalmente, la Lipofucsina se acumula en el músculo cardíaco, hepatocitos y el tejido nervioso cerebral como parte del envejecimiento.

El diagnóstico preoperatorio del BA es muy difícil debido a que sólo puede ser diagnosticado por los hallazgos patológicos. Se ha encontrado en cerca del $10 \%$ de cortes al azar de glándulas suprarrenales y hasta en un $37 \%$ cuando se incluyen pequeños nódulos en estudios de autopsia ${ }^{1}$. El diagnóstico histológico definitivo de BA excluye malignidad, pero se requiere estudio endocrinológico completo para evaluar su funcionalidad hormonal. Aunque son lesiones suprarrenales raras, el BA se ha relacionado con hiperfuncionalidad hormonal, tales como Síndrome de Cushing y Síndrome de Conn ${ }^{11-13}$.

Cuando una lesión adrenal se encuentra en la estadificación de un cáncer pulmonar en el FDG-PET/ $\mathrm{CT}$, y ante la ausencia de marcadores de función suprarrenal alterada, puede plantearse realizar una biopsia por punción percutánea. Si la apariencia macroscópica en los fragmentos obtenidos es negra, se debe considerar como primera opción un BA. Sin embargo, existen otras lesiones con apariencia macroscópica oscura, como son el melanoma adrenal y la hemorragia suprarrenal. Sólo se ha descrito un caso de BA y cáncer pulmonar, como el caso descrito en este trabajo ${ }^{14}$.

En conclusión, el BA es un adenoma suprarrenal clínicamente benigno, muy infrecuente, pero que debe ser considerado en el diagnóstico diferencial de las masas renales, dado que puede mostrar patrones falsos-positivos de malignidad o resultados no concluyentes de benignidad con las técnicas radiológicas más modernas, incluyendo TC, MRI, Cintigrafía MIBG y FDG-PET/CT.

\section{Referencias}

1.- Young WF Jr. Management approaches to adrenal incidentalomas: a view from Rochester, Minnesota. Endocrinol Metab Clin North Am. 2000;29:159-85.

2.- Kloos RT, Gross MD, Francis IR, Korobkin M, Shapiro B. Incidentally discovered adrenal masses. Endocr Rev. 1995; 16:460-84.

3.- Gac P, Cabané P, Jans J, Marambio A, Díaz M, Araya V, y cols. Manejo quirúrgico del incidentaloma suprarrenal. Rev Chil Cir. 2012;64:25-31.

4.- Castillo O, Cortés O, Kerkebe M, Pinto I, Arellano L, Russo M. Adrenalectomía laparoscópica: lecciones aprendidas en 110 procedimientos. Rev Chil Cir. 2006;58:175-80.

5.- Castillo O, Vitagliano G, Sánchez-Salas R, Díaz M. Adrenalectomía Laparoscópica. En: Cirugía Laparoscópica Urológica: Trucos y Secretos, Ed. Galaxia, Venezuela, 2006, pág. 1-12.

6.- Castillo O, Cortés O, Kerkebe M, Pinto I, Arellano L, Contreras M. Laparoscopic surgery in the treatment of adrenal pathology: experience with 200 cases. Actas Urol Esp. 2006;30:926-32.

7.- Nakajo M, Nakajo M, Kajiya Y, Tani A, Tsuruta M, Sugita S, et al. A black adrenal adenoma difficult to be differentiated from a malignant adrenal tumor by CT, MRI, scintigraphy and FDG PET/CT examinations. Ann Nucl Med. 2011;25:812-7.

8.- Mayo-Smith WW, Boland GW, Noto RB, Lee MJ. State-of the-art adrenal imaging. RadioGraphics 2001;21:995-1012.

9.- Gupta NC, Graeber GM, Tamim WJ, Rogers JS, Irisari 
L, Bishop HA. Clinical utility of PET-FDG imaging in differentiation of benign from malignant adrenal masses in lung cancer. Clin Lung Cancer 2001;3:59-64.

10.- Grumbach MM, Biller BM, Braunstein GD, Campbell KK, Carney JA, Godley PA, et al. Management of the clinically inapparent adrenal mass ("incidentaloma"). Ann Intern Med. 2003;138:424-9.

11- Armand R, Cappola AR, Horenstein RB, Drachenberg CB, Sasano H, Papadimitriou JC. Adrenal cortical adenoma with excess black pigment deposition, combinedwith myelolipoma and clinical Cushing's syndrome. Int J Surg Pathol. 2004;12:57-61.
12.- Komiya I, Takasu N, Aizawa T, Yamada T, Koizumi Y, Hashizume K, et al. Black (or brown) adrenal cortical adenoma: its characteristic features on computed tomography and endocrine data. J Clin Endocrinol Metab. 1985;61:711-7.

13.- Caplan RH, Virata RL. Functional black adenoma of the adrenal cortex: a rare cause of primary aldosteronism. Am J Clin Pathol. 1974;62:97-103.

14.- Prince EA, Yoo DC, DeLellis RA, Mayo-Smith WW. CT and PET appearance of a pigmented "black" adrenal adenoma in a patient with lung cancer. Clin Radiol. 2007;62:1229-31 